\title{
Whole Blood or Product Procurement
}

National Cancer Institute

\section{Source}

National Cancer Institute. Whole Blood or Product Procurement. NCI Thesaurus. Code C133294.

The procurement of whole blood or blood products for transfusion or further manufacturing into injectable or non-injectable products. 